\title{
Interference Minimization Resource Allocation for V2X Communication Underlaying 5G Cellular Networks
}

\author{
Xiaoqin Song, ${ }^{1}$ Kuiyu Wang, ${ }^{1}$ Lei Lei $\mathbb{D},{ }^{1}$ Liping Zhao, ${ }^{2}$ Yong $\mathrm{Li}^{2}$ and Jiankang Wang ${ }^{3}$ \\ ${ }^{1}$ College of Electronic and Information Engineering, Nanjing University of Aeronautics and Astronautics, Nanjing, China \\ ${ }^{2}$ The 63rd Research Institute of National University of Defense Technology, China \\ ${ }^{3}$ Samsung Research China-Beijing (SRC-B), Beijing, China \\ Correspondence should be addressed to Lei Lei; leilei@nuaa.edu.cn
}

Received 22 January 2020; Accepted 4 May 2020; Published 4 September 2020

Academic Editor: Xingwang Li

Copyright ( 2020 Xiaoqin Song et al. This is an open access article distributed under the Creative Commons Attribution License, which permits unrestricted use, distribution, and reproduction in any medium, provided the original work is properly cited.

In this paper, the resource allocation for vehicle-to-everything (V2X) underlaying 5G cellular mobile communication networks is considered. The optimization problem is modeled as a mixed binary integer nonlinear programming (MBINP), which minimizes the interference to $5 \mathrm{G}$ cellular users (CUs) subject to the quality of service (QoS), the total available power, the interference threshold, and the minimal transmission rate. To achieve that, the original MBINP is decomposed into three steps: transmission power initialization, subchannel assignment, and power allocation. Firstly, the minimum transmission power required by the V2X users (VUs) is set as the initial power value. Secondly, the Hungarian algorithm is used to obtain the appropriate subchannel. Finally, an optimization mechanism is proposed to the power allocation. Simulation results show that the proposed algorithm can not only ensure the minimal transmission rate of VUs but also further improve the CUs' channel capacity under the premise of guaranteeing the QoS of the CUs.

\section{Introduction}

Nowadays, vehicle-to-everything (V2X) communication [1], an important main area of Internet of things (IoT) in the intelligent transportation systems (ITS), has attracted considerable attention. It is anticipated that there will be 1.5 billion mobile-connected devices in 2020 [2], including a large number of IoT devices.

On the other hand, 3GPP is working with the cellular V2X based on the new radio (NR) of the fifth-generation (5G) cellular mobile communication networks in release 16 [3]. It is one of typical applications of ultrareliability low-latency communications (uRLLC) in 5G [4] and can extensively provide enhanced V2X scenarios, such as vehicle platform, advanced driving, extended sensors, and remote driving [5]

It is expected that the future V2X communication will offer a wide range of services, such as safety applications, road traffic management, mobile video streaming, and other entertainment applications. This poses a greater challenge to the reasonable allocation of radio resources for $\mathrm{V} 2 \mathrm{X}$ communication, especially to improve the channel capacity on the premise of ensuring the communication quality of the cellular users (CUs) [6].

There are two main spectrum sharing modes, namely, the underlay and the overlay spectrum access [7]. In the underlay mode, the V2X users (VUs) and the CUs can use the same licensed spectrum at the same time, but due to simultaneous access, the VUs inevitably cause the cochannel interference to the CUs. Therefore, it is necessary to control the interference of the VUs to the CUs so that the VUs can access the licensed spectrum under the condition of guaranteeing the quality of service (QoS) of the CUs. However, in the overlay mode, the VUs and the CUs also share the licensed spectrum at the same time. Different from the underlay mode, the VUs need the relevant information of the CUs, so as to help the CUs to send some information to compensate for their interference to the CUs, and then use the remaining time slots to transmit their own data.

This paper focuses on the underlay mode. The V2X communication [8] underlaying $5 \mathrm{G}$ cellular network means that the closer VUs can bypass the $5 \mathrm{G}$ base station (gNB) and establish direct communication pairs to each other. The 


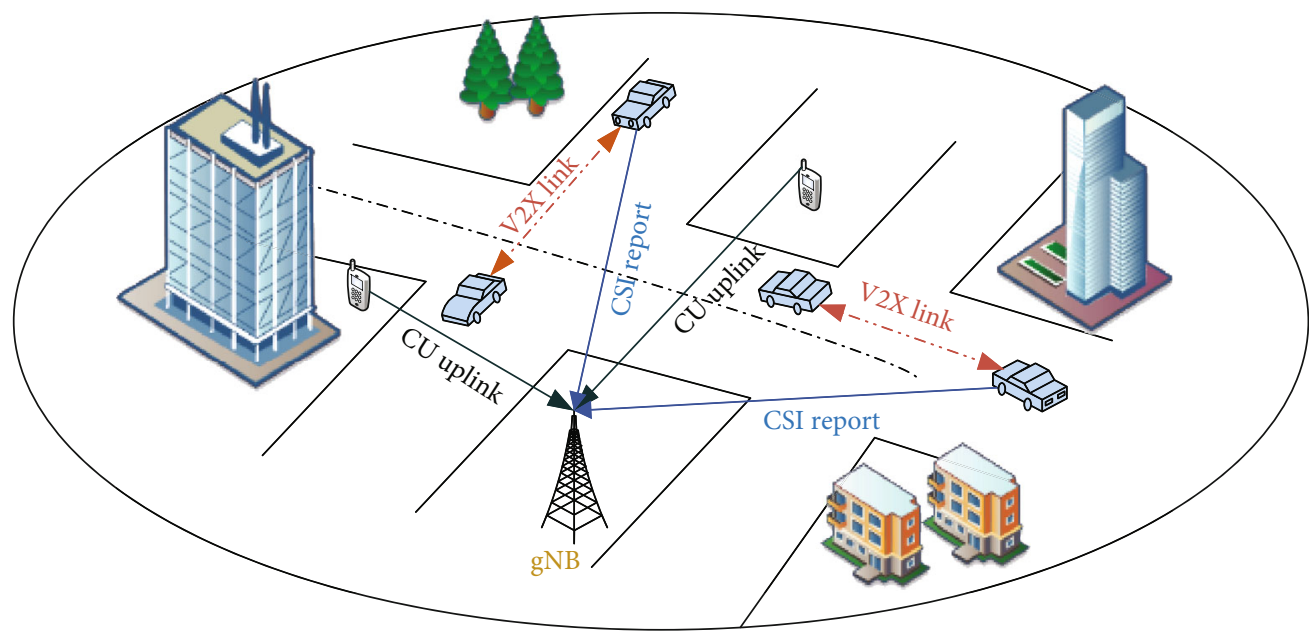

FIGURE 1: System model of underlaying V2X communication in 5G cellular network.

VUs can transmit on the cellular spectrum subject to the tolerable interference to the CUs. Therefore, the underlaying V2X communication can not only offload traffic from the $5 \mathrm{G}$ cellular infrastructure $[9,10]$ but also improve the spatial frequency reuse and energy efficiency.

To handle the ever-increasing demand of user associations, resource allocation for V2X communication underlaying 5G cellular networks [11] has attracted significant research and industry interests. In [12], a model for 5G virtualized networks in a heterogeneous cloud infrastructure is proposed, and resource allocation is formulated as a convex optimization problem, maximizing the overall system utility function. Halabian proposed a joint resource allocation solution that considers a double-sided auction-based distributed resource allocation (DS-ADRA) method [13]. Li et al. propose a method to maximize the sum of the proportional fairness functions of all users, while simultaneously taking into account factors such as the fairness, signal to interference plus noise ratio (SINR) requirements, and severe interference to ensure the QoS of users [14]. In [15], a resource allocation framework is presented for spectrum underlay in cognitive wireless networks and taking into account both interference constraints for primary users and quality of service (QoS) constraints for secondary users. In [16], a low-complexity algorithm is proposed to solve the nonconvex problem. Candidate D2D user equipment (DUE) sets are narrowed down according to required outage probability constraints, which are used to construct a simplified bipartite graph and then the Hungarian algorithm is used to determine the optimal pairing between D2D links and cellular user equipment (CUE). Zhang et al. propose a distance-based power control scheme for D2D communication underlaying uplink cellular network to achieve the expected performance gain without generating evident interference to primary CUE [17].

In this paper, our optimization objective is to minimize the interference to $5 \mathrm{G}$ CUs considering the SNR and rate requirements of VUs. The rest of the paper is organized as follows. The system model of underlay spectrum access is described in Section 2. We formulate the interference minimization underlay resource allocation problem of the described system in Section 3. Section 4 decomposes the proposed resource allocation scheme into subchannel assignment and power allocation and a suboptimal scheme is proposed. Finally, performance evaluation and conclusion are provided in Section 5 and Section 6, respectively.

\section{System Model}

We present the system model of V2X underlay $5 \mathrm{G}$ communication system, where multiple VUs coexist with multiple CUs in the same cell.

In our scheme, we only focus on the resource allocation for reusing the cellular uplink radio resources. A centralized resource allocation architecture is considered, where the gNB determines the radio resource allocation. As shown in Figure 1, if one VU (transmitter) has its designated VU (receiver) within its transmission range, the VU (transmitter) is allowed to bypass the gNB and communicates directly to the VU (receiver). This short-range, low-power V2X links coexist with the CU uplinks. The gNB has the capability of estimating the channel state information (CSI) of the cellular uplinks of CUs, whereas the CSI of the V2X links should be estimated at the VUs, then fed back to the gNB and use the uplink idle band to transmit the CSI report.

Each subchannel resource is assumed to be occupied by at most one VU link and one CU uplink. The total uplink system bandwidth $B$ of CUs is divided into $Z$ subchannels, and each VU link uses one subchannel. The index sets of subchannels are $\mathscr{Z}=\{1,2, \cdots, Z\}$. The bandwidth of each subchannel is $B_{0}=B / Z$. It is supposed that there are $L$ CUs and $K$ VUs, and the index sets of the CU uplinks and VU links are $\mathscr{L}=\{1,2, \cdots, L\}$ and $K=\{1,2, \cdots, K\}$, respectively.

\section{Problem Formulation}

The sum of the interference caused by VUs to CUs is expressed as

$$
\sum_{l=1}^{L} I_{l}=\sum_{k=1}^{K} \sum_{z=1}^{Z} \rho_{k, z} I_{k, z}^{S},
$$


where $I_{k, z}^{S}$ denotes the interference power of the VU $k$ to a certain CU uplink on the subchannel $z \cdot \rho_{k, z}$ indicates whether a VU $k$ occupies the subchannel $z$, and $\rho_{k, z}=1$ indicates that the subchannel $z$ is occupied by the VU $k$; $\rho_{k, z}=0$ indicates that the subchannel $z$ is not occupied by the VU $k$. Each subchannel can be occupied by only one $\mathrm{VU}$ at most.

The interference that the CUs can tolerate should satisfy

$$
\mathrm{C} 1: I_{k, z}^{S} \leq I^{\text {th }}, \quad \forall k \in \mathscr{K}, z \in \mathscr{Z},
$$

where $I^{\text {th }}$ is the interference tolerance threshold of the CUs.

To verify the QoS of the VUs, the SINR should satisfy

$$
\mathrm{C} 2: \operatorname{SINR}_{k, z}^{S} \geq \vartheta_{k}^{\text {th }}, \quad \forall k \in \mathscr{K}, z \in \mathscr{Z},
$$

where $\vartheta_{k}^{\text {th }}$ is the SINR threshold of the VU.

The transmission rate of each VU link should satisfy

$$
\text { C3: } R_{k} \geq R_{\min }, \quad \forall k \in \mathscr{K},
$$

where $R_{\min }$ is the minimum transmission rate of the VU.

The power constraint of the VU $k$ should satisfy

$$
\text { C4 : } \sum_{z=1}^{Z} \rho_{k, z} P_{k, z}^{S} \leq P_{k}, \quad \forall k \in \mathscr{K},
$$

where $\rho_{k, z}$ can be either 1 or 0 to represent whether the subchannel $z$ is occupied by the $k$ th VU or not; $P_{k}$ is the maximum transmission power budgets of the $\mathrm{VU} k$.

The optimization goal is to minimize the sum of the interference caused by the VUs to CUs. According to the above analysis, the objective function is expressed as

$$
\begin{gathered}
\min \sum_{k=1}^{K} \sum_{z=1}^{Z} \rho_{k, z} I_{k, z}^{S} \\
\text { s.t.C1 C4 } \\
\text { C5 }: \rho_{k, z} \in\{0,1\}, \quad \forall k \in \mathscr{K}, z \in \mathscr{Z} \\
\text { C6 }: \sum_{k=1}^{K} \rho_{k, z} \leq 1, \quad \forall z \in \mathscr{Z},
\end{gathered}
$$

where C5 and C6 indicate that each subchannel is at most used by one VU.

\section{The Proposed Allocation Algorithm}

In order to facilitate the solution, we first transform the initial objective function (6) into an equivalent function. First, according to the SINR formula

$$
\operatorname{SINR}_{k, z}^{S}=\frac{P_{k, z}^{S} G_{k, z}^{S}}{I_{l, z}^{O}}
$$

where $P_{k, z}^{S}$ is the transmission power; $G_{k, z}^{S}$ is the channel power gain value; $I_{l, z}^{O}$ is the sum of interference and noise on subchannel $z$ to the $\mathrm{CU} l$.

Next, define $Z^{\prime}$ as the total number of subchannels actually allocated to $K$ VUs; the index sets of which are $\mathscr{Z}^{\prime}=\left\{1,2, \cdots, Z^{\prime}\right\}$. It is reasonable to assume that the number of subchannels that the network can provide is sufficient, that is, $Z^{\prime} \leq Z$. $Z^{\prime}$ can be expressed as

$$
Z^{\prime}=\sum_{k=1}^{K} \frac{R_{k}}{B_{0} \log _{2}\left(1+\operatorname{SINR}_{k}^{S}\right)},
$$

where $\operatorname{SINR}_{k}^{S}$ is the minimum SINR among the subchannels actually used by the $\mathrm{VU} k$.

We consider that the SINR of the CUs in the system must also be satisfied. If we let $\beta^{*}$ as the minimum SINR threshold of the CU $l$, the maximum interference threshold $I^{\text {th }}$ on the actually allocated subchannel $z^{\prime}$ of the $\mathrm{CU}$ receiver can be determined by

$$
I^{\mathrm{th}}=\frac{g_{l, z^{\prime}}^{P} P_{l, z^{\prime}}^{P}}{\beta^{*}}-N_{0} B_{0}
$$

where $N_{0}$ is the noise power spectral density.

In order to minimize the interference caused by the VUs to the CUs, when the subchannel allocation is fixed, the SINR and data transmission rate of the VUs must be as low as possible. Therefore, the QoS of the VU resource allocation should take the minimum threshold, that is,

$$
\begin{aligned}
& \operatorname{SINR}_{k}^{S}=\vartheta_{k}^{\text {th }}, \\
& R_{k}=R_{\text {min }} .
\end{aligned}
$$

When (10) and (11) are satisfied, the number of subchannels $m_{k}$ allocated by the gNB to the VU $k$ can be expressed as

$$
m_{k}=\left\lceil\frac{R_{\min }}{B_{0} \log _{2}\left(1+\vartheta_{k}^{\text {th }}\right)}\right\rceil .
$$

And (8) can be rewritten as

$$
Z^{\prime}=\sum_{k=1}^{K} m_{k}=\sum_{k=1}^{K} \frac{R_{\min }}{B_{0} \log _{2}\left(1+\vartheta_{k}^{\text {th }}\right)} .
$$

The interference constraints and SINR constraints of the original optimization problem can be expressed as

$$
\mathrm{C} 1^{\prime}: \frac{I_{l, z^{\prime}}^{o} \vartheta_{k}^{\text {th }}}{G_{k, z^{\prime}}^{S}} \leq P_{k, z^{\prime}}^{S} \leq \frac{g_{l, z^{\prime}}^{P} P_{l, z^{\prime}}^{P} .}{g_{k, z^{\prime}}^{S} \beta^{*}} .
$$

The power and rate constraints in the original problem can be represented by $\mathrm{C}^{\prime}$ and $\mathrm{C}^{\prime}$, respectively. 


$$
\begin{aligned}
\mathrm{C} 2^{\prime}: \sum_{k=1}^{K} \sum_{z^{\prime}=1}^{Z^{\prime}} P_{k, z^{\prime}}^{S} & \leq P_{\mathrm{T}}, \\
\mathrm{C} 3^{\prime}: \sum_{z^{\prime}=1}^{Z^{\prime}} \rho_{k, z^{\prime}} & =\frac{R_{\min }}{B_{0} \log _{2}\left(1+9_{k}^{\text {th }}\right)} .
\end{aligned}
$$

According to the analysis above, the equivalent form of the original optimization problem in (6) is expressed as

$$
\begin{gathered}
\min \sum_{k=1}^{K} \sum_{z^{\prime}=1}^{Z^{\prime}} \rho_{k, z^{\prime}} I_{k, z^{\prime}}^{S} \\
\text { s.t.C1 } 1^{\prime} \sim \mathrm{C} 3^{\prime} \\
\mathrm{C} 4^{\prime}: \nabla \rho_{k, z^{\prime}} \in\{0,1\}, \quad \forall k \in \mathscr{K}, z^{\prime} \in \mathscr{Z}^{\prime} \\
\mathrm{C} 5^{\prime}: \otimes \sum_{k=1}^{K} \rho_{k, z^{\prime}} \leq 1, \quad \forall z^{\prime} \in \mathscr{Z}^{\prime} .
\end{gathered}
$$

Obviously, the optimization problem proposed in this paper is a mixed binary integer nonlinear programming (MBINP) problem. The variables to be solved are integer variable $\rho_{k, z^{\prime}}$ and continuous variable $P_{k, z^{\prime}}^{S}$, which are difficult to solve directly. Therefore, we use a stepwise algorithm for subchannel and power allocation. The main process of the algorithm includes three steps. First, the VU transmit power is initialized; then, when the power is determined, the Hungarian algorithm is used to complete the subchannel allocation; finally, power allocation is performed, and the initialization power of the subchannel allocated on the VU is limited according to the interference constraint condition and the power constraint condition. If these constraints are not satisfied, the VU on the subchannel refuses to share the frequency spectrum with the CU. The specific process of the algorithm is described as follows.

4.1. Transmit Power Initialization. Since the optimization goal is to minimize interference, according to equation $I_{k, z^{\prime}}^{S}$ $=g_{k, z^{\prime}}^{S} P_{k, z^{\prime}}^{S}$, interference is equal to the channel gain of the interfering link multiplied by the transmission power, so the transmission power of the VUs is also required to be minimal. Under constraint $\mathrm{Cl}^{\prime}$, suppose

$$
\frac{I_{l, z^{\prime}}^{o} \vartheta_{k}^{\text {th }}}{G_{k, z^{\prime}}^{S}} \leq \frac{g_{l, z^{\prime}}^{P} P_{l, z^{\prime}}^{P}}{g_{k, z^{\prime}}^{S} \beta^{*}},
$$

where $g_{k, z}^{S}$ is the power gain on the interference link from the cognitive base station to the receiving end of the CU, $g_{l, z^{\prime}}^{P}$ is the power gain of the CU to the VU on the interference link, and $P_{l, z^{\prime}}^{P}$ is the transmission power of the CU $l$ on the subchannel $z^{\prime}$.

If (17) holds and constraint condition $\mathrm{C}^{\prime}{ }^{\prime}$ is satisfied, then the minimum transmission power required by the $\mathrm{VU}$ $k$ on the subchannel $z^{\prime}$ is expressed as
TABLE 1: Simulation parameters.

\begin{tabular}{lc}
\hline Parameter & Value \\
\hline gNB coverage radius $(R)$ & $500 \mathrm{~m}$ \\
Number of CUs $(L)$ & 4 \\
Number of VUs $(K)$ & $2,4,8,16,32$ \\
Subchannel bandwidth $\left(B_{0}\right)$ & $0.3215 \mathrm{MHz}$ \\
Pathloss factor $(\alpha)$ & 4 \\
Noise power spectral density $\left(N_{0}\right)$ & $4 \times 10^{-20} \mathrm{~W} / \mathrm{Hz}$ \\
Transmission power of CU $\left(P_{l, z}^{P}\right)$ & $100 \mathrm{~mW}$ \\
Total transmission power of gNB $\left(P_{\mathrm{T}}\right)$ & $2 \mathrm{~W}$ \\
SINR of CUs $\left(\beta^{*}\right)$ & $20 \mathrm{~dB}$ \\
SINR of VUs $\left(\vartheta_{k}^{\text {th }}\right)$ & {$[-15 \mathrm{~dB}, 15 \mathrm{~dB}]$} \\
\hline
\end{tabular}

$$
P_{k, z^{\prime}}^{S}=\frac{I_{l, z^{\prime}}^{o} \vartheta_{k}^{\text {th }}}{G_{k, z^{\prime}}^{S}} .
$$

4.2. Subchannel Allocation. After completing the power initialization, the optimization problem can be expressed as

$$
\begin{gathered}
\min _{\rho_{k, z}} \sum_{k=1}^{K} \sum_{z^{\prime}=1}^{Z^{\prime}} \rho_{k, z^{\prime}} \frac{g_{k, z^{\prime}}^{S} I_{l, z^{\prime}}^{o} 9_{k}^{\text {th }}}{G_{k, z^{\prime}}^{S}} \\
\text { s.t.C3' }: \sum_{z^{\prime}=1}^{Z^{\prime}} \rho_{k, z^{\prime}}=\frac{R_{\min }}{B_{0} \log _{2}\left(1+\vartheta_{k}^{\text {th }}\right)} \\
\mathrm{C} 4^{\prime}: \rho_{k, z^{\prime}} \in\{0,1\}, \quad \forall k \in \mathscr{K}, z^{\prime} \in \mathscr{Z}^{\prime} \\
\mathrm{C} 5^{\prime}: \sum_{k=1}^{K} \rho_{k, z^{\prime}} \leq 1, \quad \forall z^{\prime} \in \mathscr{Z}^{\prime} .
\end{gathered}
$$

According to equation (19), it can be seen that the optimization problem is a $0-1$ assignment integer linear programming problem about $\rho_{k, z}$. The Hungarian algorithm [18] is used here to solve the global optimal solution of the problem.

4.3. Power Allocation. The Hungarian algorithm is used to complete the subchannel allocation. However, in order to perform the optimal power allocation, both the constraint condition $\mathrm{C}^{\prime}{ }^{\prime}$ and condition (17) assumed when initializing the power need to be reconsidered based on the subchannel allocation results. Therefore, after each VU has assigned a subchannel, power detection needs to be performed according to the constraints. If the power allocated by the VU satisfies condition (17) and the total transmission power satisfies the constraint condition $\mathrm{C} 2{ }^{\prime}$, then the power allocation result is the optimal solution of the original optimization problem and completes resource allocation; otherwise, the following VU access control iterative algorithm will be used to reallocate power.

Step 1. If assumption (17) is not satisfied, it means that the VU cannot obtain enough transmission power to satisfy its 


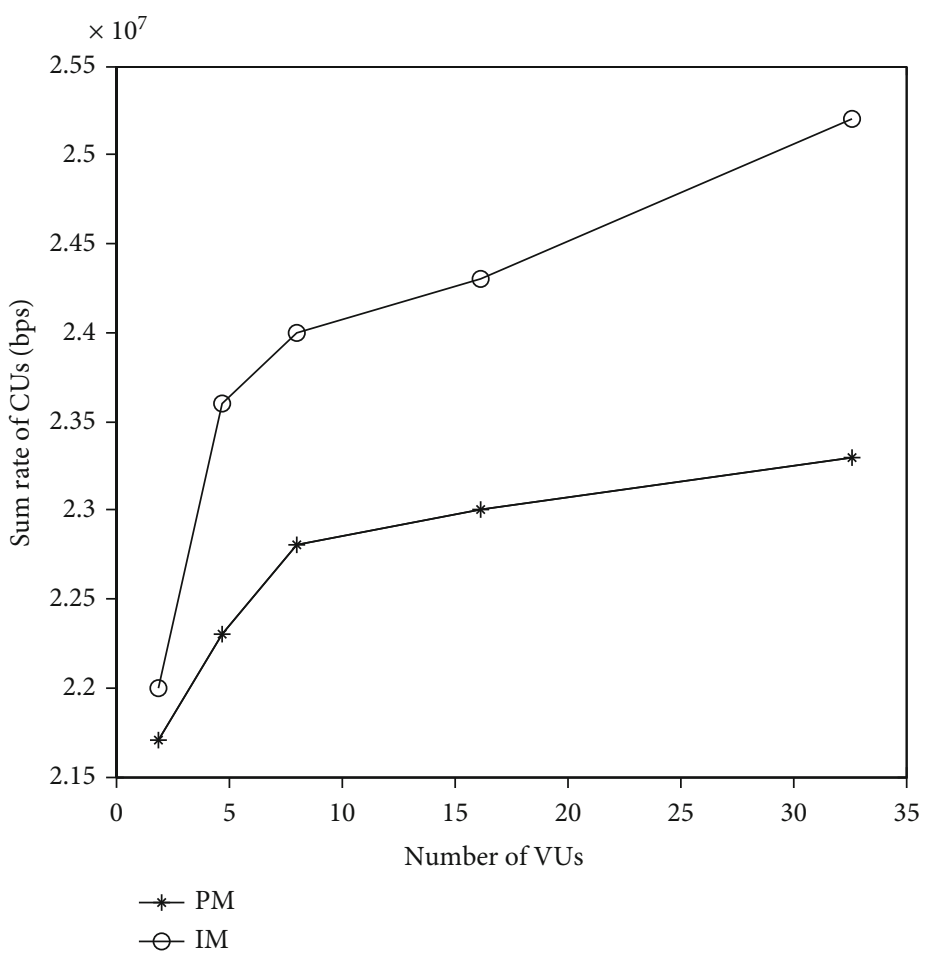

Figure 2: Performance of the number of VUs and the CU rate.

SINR requirements under the restriction of the CU interference threshold, so the VU must give up access to the shared spectrum opportunity.

Step 2. On the basis of Step 1, the VU does not give up the opportunity to access the spectrum, so the VU can choose to compromise on the data rate requirement and access the spectrum at a data rate lower than its own requirement.

Step 3. Next, considering constraint condition $\mathrm{C}^{\prime}$, the total transmission power can be obtained based on the initialization power and subchannel allocation above. If the total power exceeds the power threshold in constraint condition $\mathrm{C}^{\prime}$ ', the subchannel allocated to the maximum power is denied access to the VU. Then, recalculate the power until the constraint condition $\mathrm{C}^{\prime}$ is satisfied, at which point the power allocation is completed.

\section{Performance Evaluation}

This section uses MATLAB to simulate and analyze the above resource allocation algorithms and compares them with traditional algorithms. The simulation results are the average of 1,000 Monte Carlo experiments. The simulation parameters are shown in Table 1. One gNB and multiple VUs are considered, and all users are randomly distributed in the covered cell. The slow fading in the system model is assumed to be multipath Rayleigh fading, pathloss factor $\alpha$ $=4$, and shadow fading standard deviation $\sigma_{X Y}=4 \mathrm{~dB}$. It is also assumed that the angle between the movement direction of each VU and the horizontal axis of the coordinate system is a random value uniformly distributed between 0 and $2 \pi$. Simultaneously, the location information and spectrum sensing results of the VUs can be transmitted to the gNB through dedicated channels. According to [19], the authors propose that in a mobile scenario and the VUs predict the channel state information, assuming that the time required for the VUs to sense once is $1 \mathrm{~ms}$, then the time for the VUs to sense twice is $2 \mathrm{~ms}$.

We first analyze the complexity of the algorithm. The complexity of the proposed resource allocation algorithm is determined by three steps in Section 3. Due to the small number of subchannels and VUs, compared with the integer programming problem in the second step, the complexity of the initialization power in the first step and the iterative power allocation in the third step is greatly reduced and can be ignored. So the complexity of the resource allocation algorithm mainly depends on the second step of the subchannel allocation process. According to [20], the complexity of the Hungarian subchannel allocation algorithm is $O\left(K^{3} Z^{\prime 3}\right)$.

In order to facilitate the analysis and ensure the fairness of the comparative analysis, first define the algorithm for comparison. For the comparison of power minimization algorithms, we consider the same constraints and solution algorithms, but change the optimization goal to minimize the transmission power of VUs, it is expressed as "PM." The complexity of $\mathrm{PM}$ is also $O\left(K^{3} Z^{\prime 3}\right)$. Meanwhile, the minimum interference we propose is expressed as "IM."

Figures 2 and 3 compare the two algorithms, showing the rate of the CUs, and the performance of the total power required by the VUs varies with the number of VUs. 


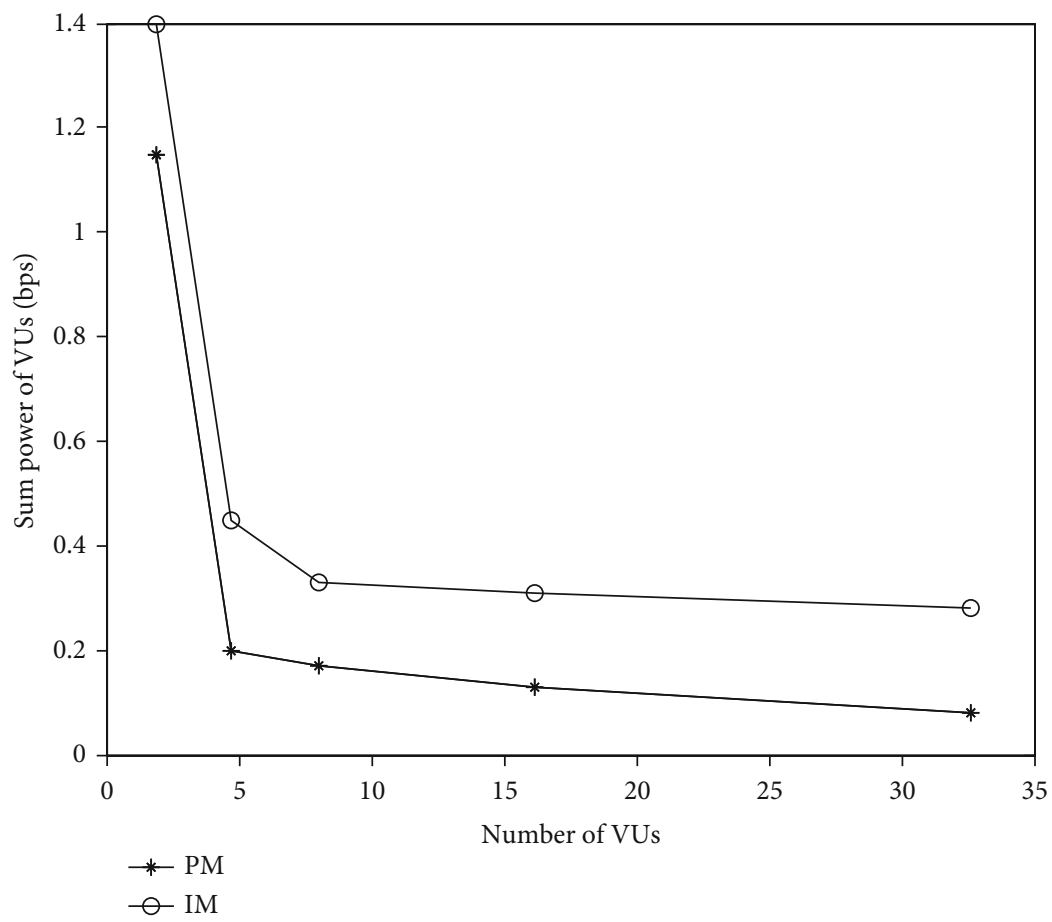

FIgURE 3: Performance of the number of VUs vs. the total power of VUs.

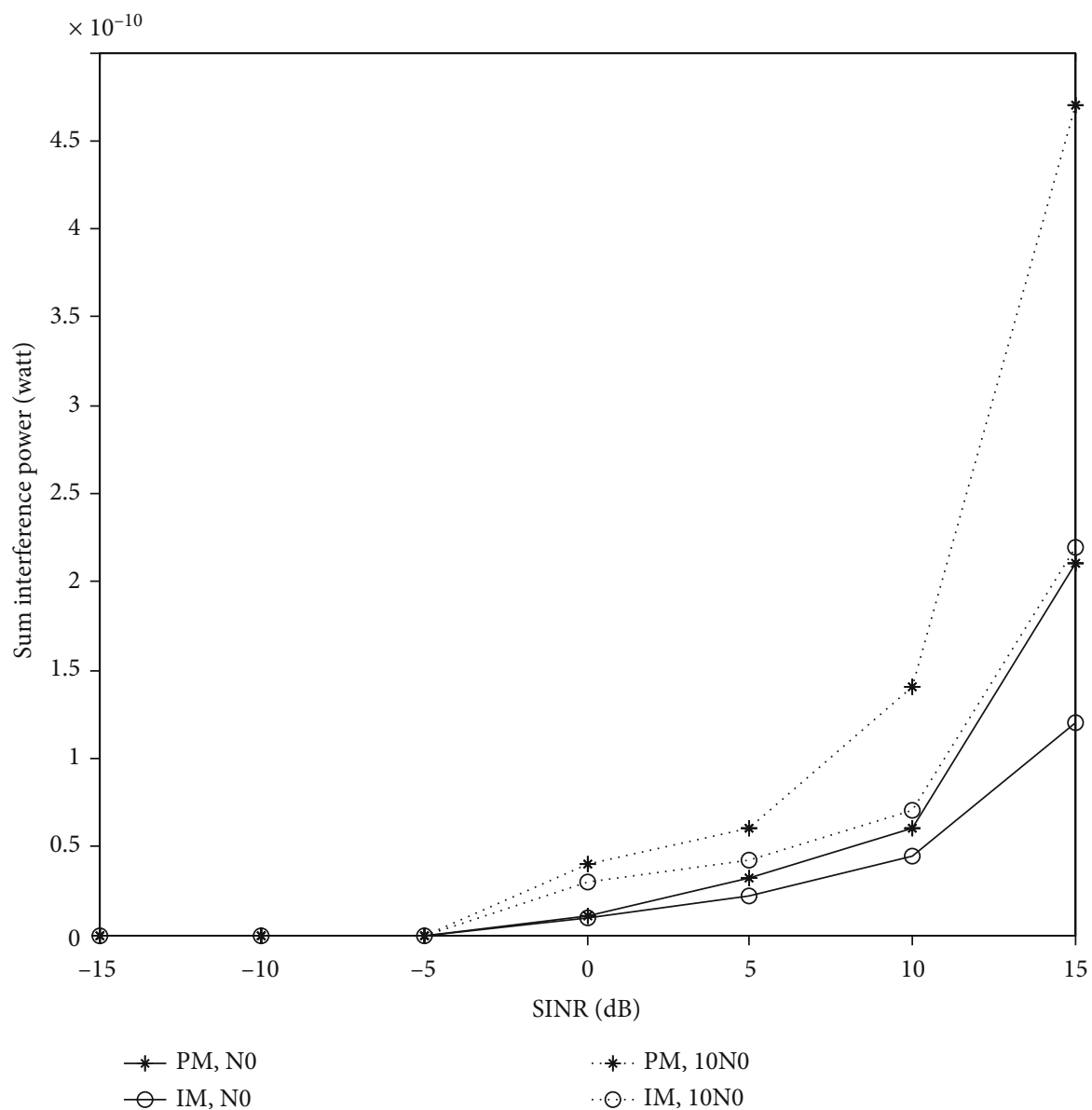

FIGURE 4: Performance of SINR, noise power, and interference of CUs. 


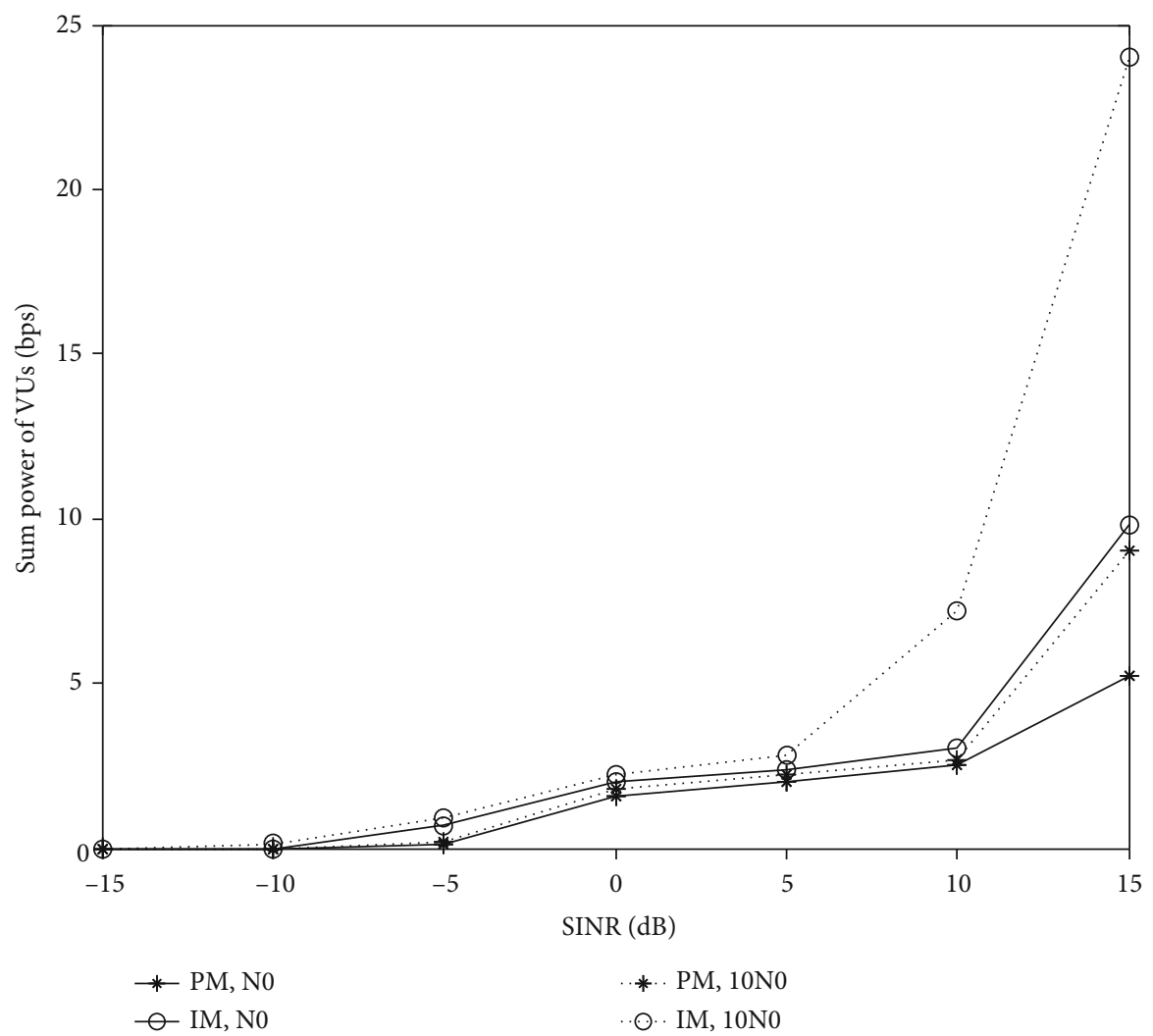

Figure 5: Performance of SINR, noise power, and total power of VUs.

The CU rate obtained by the PM and IM algorithms increases with the number of VUs, and under the same conditions, the IM algorithm can obtain a larger CU rate in Figure 2. This is also because when the number of VUs increases, the subchannels have more opportunities to be assigned to better channel states and VUs with less interference, and when the CUs suffer less interference, the CU rate will inevitably increase.

Total power of the VUs obtained by the IM and PM algorithms decreases as the number of VUs increases in Figure 3. When the number of VUs is small, the cognitive base station total power required by the VUs is large. However, as the number of VUs increases, subchannels can be allocated to more suitable VUs, so the required power is naturally reduced.

Considering Figures 2 and 3 together, it can be seen that the conclusions obtained by the two figures are basically the same: the proposed IM algorithm not only guarantees QoS, power, and interference of VUs but also reduces the interference to CUs, thus protecting the capacity of CUs and improving the adaptability of CUs to different business requirements.

Figures 4 and 5 compare and analyze the rules of the interference to the CUs and the performance of the total power required by the VUs with the change of the SINR of the VUs under two different noise power conditions.

As can be seen from Figure 4, the CU interference obtained by the IM and PM algorithms increases with the VU SINR. Compared with PM, IM has the least interference to the CUs. From Figure 5, we can see the same trend. When the VU SINR is relatively small, the total power obtained by IM and PM is small. However, with the increase of the SINR of the VUs, the total power of IM and PM also increases. Combining the two graphs shows that when the SINR is relatively low, the performance of the IM and PM algorithms is better. As the SINR becomes larger, the VUs require more power, and the interference to the CUs will also increase. If the SINR continues to increase, the power required by the VUs and the interference caused to the CUs will exceed the threshold value. At the same time, it can be seen that the larger the noise power, the greater the power required by the system and the interference to the CUs, and the performance is far worse than under low noise power conditions.

Finally, Figure 6 compares the performance of the two algorithms with the change of SINR of VUs. It can be seen from Figure 6 that the CU rate obtained by the IM and PM algorithms decreases as the SINR increases, and the CU rate obtained by the PM algorithm is lower than that in the IM algorithm. At the same time, the rate of the CUs is kept at an acceptable low level due to the significant increase in the rate of the VUs. For the IM and PM algorithms, as the SINR increases, cognitive base stations need to allocate more power to VUs to satisfy their requirements. Therefore, the VUs will inevitably cause greater interference to the CUs. According to the Shannon channel capacity formula of the CUs, gradually increasing interference will cause the CU rate to decrease. 


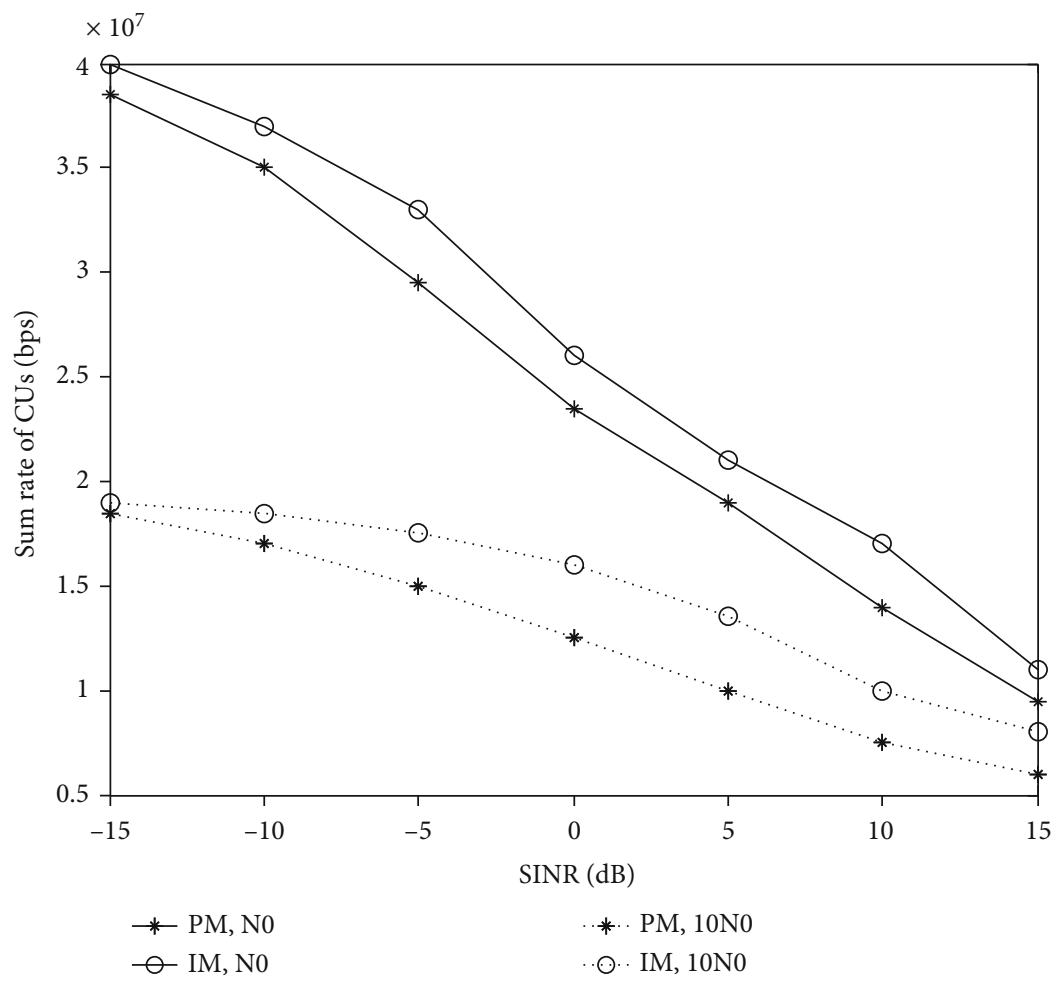

Figure 6: Performance of SINR vs. CU rate.

Combined with Figures 4-6, it can be seen that the performance of IM and PM algorithms is similar with the increase of SINR of VUs, but IM can have less interference, which further protects the channel capacity of CUs.

\section{Conclusion}

In this paper, we propose a resource allocation algorithm to V2X communication under 5G networks, where VUs and CUs coexist in $5 \mathrm{G}$ cellular networks. Considering the constraints of the SINR and the minimal rate and the interference threshold requirements of each VU, the power and subchannel allocation of the VUs is allocated according to the channel gain of the VUs and the interference of each VU link to the CUs. The objective function is established according to the goal of minimizing interference while satisfying multiple constraints. We use three stages to solve MBINP and propose an optimization power allocation mechanism. Simulation results demonstrate that, compared with PM and RM algorithms, the proposed resource allocation algorithm can satisfy the QoS of VUs while minimizing the interference to CUs and can further protect the channel capacity of the CUs.

\section{Data Availability}

The model data used to support the findings of this study are included within the article. The code data used to support the findings of this study have not been made available because the data also forms part of an ongoing study.

\section{Conflicts of Interest}

The authors declare that there is no conflict of interest regarding the publication of this paper.

\section{Acknowledgments}

This work is supported by the National Natural Science Foundation of China (No. 61572254, No. 61902182, and No. 61301103), the Natural Science Foundation of Jiangsu Province of China (No. BK20161488), the Aeronautical Science Foundation of China (No. 2016ZC52029), the Qing Lan Project of Jiangsu Province of China, China Postdoctoral Science Foundation (No. 2019TQ0153), and the Foundation of CETC Key Laboratory of Aerospace Information Applications of China (No. SXX18629T022).

\section{References}

[1] S. Chen, J. Hu, Y. Shi et al., "Vehicle-to-everything (v2x) services supported by LTE-based systems and 5G," IEEE Communications Standards Magazine, vol. 1, no. 2, pp. 70-76, 2017.

[2] M. Peng, Y. Sun, X. Li, Z. Mao, and C. Wang, "Recent advances in cloud radio access networks: system architectures, key techniques, and open issues," IEEE Communications Surveys \& Tutorials, vol. 18, no. 3, pp. 2282-2308, 2016.

[3] Study on NR Vehicle-to-Everything (V2X), Standard 3GPP TR 38.885, 2018.

[4] X. Li, J. Li, Y. Liu, Z. Ding, and A. Nallanathan, "Residual transceiver hardware impairments on cooperative NOMA networks," IEEE Transactions on Wireless Communications, vol. 19, no. 1, pp. 680-695, 2020. 
[5] Y. Kim, F. Sun, Y. Wang et al., "New radio (NR) and its evolution toward 5G-advanced," IEEE Wireless Communications, vol. 26, no. 3, pp. 2-7, 2019.

[6] N. Cheng, H. Zhou, L. Lei et al., "Performance analysis of vehicular device-to-device underlay communication," IEEE Transactions on Vehicular Technology, vol. 66, no. 6, pp. 5409-5421, 2017.

[7] I. de la Iglesia, U. Hernandez-Jayo, E. Osaba, and R. Carballedo, "Smart bandwidth assignation in an underlay cellular network for internet of vehicles," Sensors, vol. 17, no. 10, p. 2217, 2017.

[8] A. Asadi, Q. Wang, and V. Mancuso, "A survey on device-todevice communication in cellular networks," IEEE Communications Surveys \& Tutorials, vol. 16, no. 4, pp. 1801-1819, 2014.

[9] X. Li, J. Li, and L. Li, "Performance analysis of impaired SWIPT NOMA relaying networks over imperfect Weibull channels," IEEE Systems Journal, vol. 14, no. 1, pp. 669-672, 2020.

[10] X. Li, M. Liu, C. Deng, P. T. Mathiopoulos, Z. Ding, and Y. Liu, "Full-duplex cooperative NOMA relaying systems with I/Q imbalance and imperfect SIC," IEEE Wireless Communications Letters, vol. 9, no. 1, pp. 17-20, 2020.

[11] Q. Chen, H. Jiang, and G. Yu, "Service oriented resource management in spatial reuse-based C-V2X networks," IEEE Wireless Communications Letters, vol. 9, no. 1, pp. 91-94, 2020.

[12] L. Ferdouse, A. Anpalagan, and S. Erkucuk, "Joint communication and computing resource allocation in $5 \mathrm{G}$ cloud radio access networks," IEEE Transactions on Vehicular Technology, vol. 68, no. 9, pp. 9122-9135, 2019.

[13] H. Halabian, "Distributed resource allocation optimization in $5 \mathrm{G}$ virtualized networks," IEEE Journal on Selected Areas in Communications, vol. 37, no. 3, pp. 627-642, 2019.

[14] X. Li, R. Shankaran, M. A. Orgun, G. Fang, and Y. Xu, "Resource allocation for underlay D2D communication with proportional fairness," IEEE Transactions on Vehicular Technology, vol. 67, no. 7, pp. 6244-6258, 2018.

[15] L. Le and E. Hossain, "Resource allocation for spectrum underlay in cognitive radio networks," IEEE Transactions on Wireless Communications, vol. 7, no. 12, pp. 5306-5315, 2008.

[16] L. Wang, H. Tang, H. Wu, and G. L. Stüber, "Resource allocation for D2D communications underlay in Rayleigh fading channels," IEEE Transactions on Vehicular Technology, vol. 66, no. 2, pp. 1159-1170, 2017.

[17] Z. Zhang, R. Q. Hu, and Y. Qian, "D2D communication underlay in uplink cellular networks with distance based power control," in 2016 IEEE International Conference on Communications (ICC), pp. 1-6, Kuala Lumpur, December 2016.

[18] H. W. Kuhn, "The Hungarian method for the assignment problem," Naval Research Logistics, vol. 52, no. 1, pp. 7-21, 2005.

[19] D. W. K. Ng, E. S. Lo, and R. Schober, "Energy-efficient resource allocation in multi-cell OFDMA systems with limited backhaul capacity," IEEE Transactions on Wireless Communications, vol. 11, no. 10, pp. 3618-3631, 2012.

[20] L. Zhang, M. Xiao, G. Wu, S. Li, and Y.-C. Liang, "Energyefficient cognitive transmission with imperfect spectrum sensing," IEEE Journal on Selected Areas in Communications, vol. 34, no. 5, pp. 1320-1335, 2016. 